Bio - grafia. Escritos sobre la Biología y su Enseñanza. ISSN 2027

Edición Extraordinaria. p.p. 1636-1644

Memorias del VIII Encuentro Nacional de Experiencias en Enseñanza de la Biología y la Educación Ambiental. III Congreso Nacional de Investigación en Enseñanza de la Biología.

\title{
IMPLEMENTACIÓN DE UNA UNIDAD DIDÁCTICA PARA LA ENSEÑANZA DEL CONCEPTO HERENCIA EN JÓVENES DE OCTAVO DEL COLEGIO MANUELITA SÁENZ IED.
}

\section{IMPLEMENTATION OF A DIDACTIC UNIT FOR TEACHING GENETIC INHERITANCE CONCEPT IN EIGHT GRADE TEENAGERS FROM COLEGIO MANUELITA SAENZ IED.}

Cindy Lorena Baracaldo Huertas ${ }^{1}$.

\section{RESUMEN}

La enseñanza del concepto de herencia es parte importante del currículo actual de ciencias naturales ya que pone de presente la explicación compleja de la transmisión de información genética de generación en generación, y éste proceso a su vez, le permite al estudiante dilucidar que los diversos elementos integrantes del proceso de herencia genética hacen parte de él y lo constituyen como ser vivo. En este sentido, este artículo pretende mostrar los efectos obtenidos en la aplicación de la unidad didáctica: "Herencia genética: una mirada a través de lo que somos" en jóvenes de octavo grado, así como también las reacciones que tuvo la profesora titular del curso durante el desarrollo de las diversas actividades propuestas en esta unidad, la cual maneja un enfoque expositivo, en donde el aprendizaje significativo es la mejor herramienta que puede utilizar el docente. Así pues, se concluye que las experiencias innovadoras constituyen una fuente importante de aprendizaje, razón por la que el docente debe tener siempre la mente abierta a cambios en la forma de construir conocimiento con los estudiantes.

PALABRAS CLAVE: Cromosoma, Gen, ADN, Conocimiento científico escolar.

${ }^{1}$ Estudiante de sexto semestre de Licenciatura en Biología de la Universidad Distrital Francisco José de Caldas.

${ }^{1}$ Estudiante de sexto semestre de Licenciatura en Biología de la Universidad Distrital Francisco José de Caldas. 1636 
Bio - grafia. Escritos sobre la Biología y su Enseñanza. ISSN 2027

Edición Extraordinaria. p.p. 1636-1644

Memorias del VIII Encuentro Nacional de Experiencias en Enseñanza de la Biología y la Educación Ambiental. III Congreso Nacional de Investigación en Enseñanza de la Biología.

\begin{abstract}
The Inheritance concept teaching is an important part in actual natural sciences curriculum, as highlights the complex explanation of genetic information between generations, at the same time this process let students elucidate that different elements which integrate inheritance process compose them and make them living beings. In this sense, this text pretends to show the effects obtained in the application of didactic unit called: "Herencia genética: una mirada a través de lo que somos" with eight grade teenagers, as well the titular proffesor's reactions during the development of different activities which were proposed in the didactid unit, thisone is based in the exhibition focus where significant learning is a wonderfull tool that teachers can use. So, it is concluded that innovative experences make an important learning source, reason why teachers should have open mind to changes in the way of making knowledgement with students.
\end{abstract}

KEY WORDS: Chromosome, Gene, DNA, Scholar scientific knowlegement.

\title{
INTRODUCCIÓN:
}


Edición Extraordinaria. p.p. 1625- 1635

Memorias del VIII Encuentro Nacional de Experiencias en Enseñanza de la Biología y la Educación Ambiental. III Congreso Nacional de Investigación en Enseñanza de la Biología.

Recientemente se han llevado a cabo estudios alrededor de la didáctica de la genética con el fin de construir redes conceptuales en torno a los conceptos básicos de la herencia que se trabajan en las escuelas y cuya enseñanza se hace difícil por la complejidad misma de los conceptos; más aún por la aplicación de las matemáticas en casi todos sus campos de trabajo (Bugallo Rodríguez, 1995). Además tal como lo mencionan Stewart y Kirk, (1990), la genética es uno de los temas más tratados en la didáctica de la biología, debido a su importancia ya que es un área de expansión con importantes implicaciones económicas, éticas y sociales; asimismo es ampliamente reconocida como la base conceptual para la comprensión de la evolución y, por lo tanto, de la propia biología (Smith \& Sims, 1992).

En este sentido, autores como Ayunso \& Banet, (2002) plantean la importancia de reestructurar la enseñanza de los conceptos de genética que en la actualidad se encuentran limitados a los ejercicios mecánicos propuestos en los libros para la educación secundaria (Ayunso, Banet, \& Abellán, 1996), -omitiendo la importancia de construir conocimientos partiendo de la discusión de las concepciones alternativas de los estudiantes (Cubero, 1994)- lo que de alguna manera limita el rol del docente en el aula, dejando de ser un guía en los procesos de construcción conceptual para convertirse en un proveedor de saberes. Esta imposibilidad de discusión presenta la genética como una ciencia terminada que debe ser memorizada porque no puede ser discutida ni modificada; de ahí la necesidad de conocer cómo se enseña la genética en la escuela, específicamente en el colegio Manuelita Sáenz I.E.D. en jóvenes de octavo grado.

Para lograrlo se realizó una charla informal -es decir un diálogo no planeado ni documentado- con la docente titular sobre la forma de abordar el tema, a lo cual la docente manifestó dar una clase teórica y posteriormente proponer el trabajo de los estudiantes con un libro de texto,-cualquiera al que el estudiante tenga acceso y que documente el tema- para disipar las inquietudes. Esto puso de presente la intención de crear una unidad didáctica en la que se abordaran los conceptos principales de la herencia genética a partir de actividades en las que se tuviera en cuenta las concepciones alternativas de los estudiantes sin dejar de lado la teoría.

La unidad didáctica Herencia genética: una mirada a través de lo que somos se construyó desde el enfoque expositivo, definido por Pozo y Gómez (2004) como un enfoque flexible que parte de las ideas previas del estudiante, cuyo fundamento es el aprendizaje significativo propuesto por Ausbel. No obstante, estos autores 
Edición Extraordinaria. p.p. 1625- 1635

Memorias del VIII Encuentro Nacional de Experiencias en Enseñanza de la Biología y la Educación Ambiental. III Congreso Nacional de Investigación en Enseñanza de la Biología.

consideran que este enfoque tiende a caer en el modelo tradicional, cosa que plantean como una desventaja porque el estudiante es percibido como una tabula raza que debe ser llenada del conocimiento que sólo posee el profesor (Pozo y Gómez, 2004). Sin embargo, para esta unidad didactica este aspecto es positivo ya que los estudiantes están acostumbrados a este modelo; pues si bien se pretendia aplicar actividades novedosas, no se queria que los estudiantes se sintieran incomodos o desconcertados con un enfonque que no comprendieran y el cual les causara dificultades a la hora de desarrollar las actividades, pues como lo mencionan Ayunso y Banet (2002):

(...) las posibilidades de innovación en el aula se asemejan a una carrera de obstáculos (...) como consecuencia de ello, una lógica prudencial obliga a reflexonar (...) antes de introducir cambios importantes en nuestra práctica educativa (Banet, 2001): - No creemos aconcejable plantear modificaciones amplias y complejas cuyo desarrollo sea dificil de controlar. Es posible que estas iniciativas no generen los resultados que esperamos (...) Por ello nuestra primera consideración se refiere al carácter gradual de estos procesos de innovación. (Ayunso \& Banet, 2002, págs. 153-154)

Además, para la construcción de la misma fueron tenidos en cuenta los Estándares de ciencias naturales establecidos por el Ministerio de Educación Nacional para el grado octavo y las competencias básicas propuestas por el ICFES.

\section{METODOLOGÍA:}

La perspectiva metodológica que se trabajó en la unidad didáctica (en adelante UD) fue la socio-crítica, ya que permite no solamente acercarse a la realidad educativa a través de la descripción y la comprensión, sino que también propone una transformación de la misma, (Mendéz Coca, 2013); pues lo que se buscaba con la aplicación de esta unidad didáctica era de alguna manera transformar la forma en la que se enseñan los conceptos básicos de la genética en el colegio Manuelita Saénz, y analizar hasta que punto estas nuevas propuestas fueron éxitosas. El método con el que se desarrollo dicha perspectiva fue el cuantitativo, implementando la técnica de encuestas con preguntas abiertas, denominadas instrumentos (para el PEI, el docente y los estudiantes), utilizando preguntas como: "¿Cuál es el impacto de la institución en la comunidad?, ¿Cuál es la cualidad más importante de la institución?" -en el caso de la encuesta aplicada a la 
Edición Extraordinaria. p.p. 1625- 1635

Memorias del VIII Encuentro Nacional de Experiencias en Enseñanza de la Biología y la Educación Ambiental. III Congreso Nacional de Investigación en Enseñanza de la Biología.

coordinadora-; ¿Cómo aborda el tema de herencia genética con los estudiantes?, ¿Cuáles son las actividades más comunes realizadas? ¿Qué tan importantes son las ideas previas de los estudiantes al iniciar un tema? ¿Qué métodos utiliza para obtener dichas ideas?,-en el caso del profesor- o ¿Cómo crees que se transmite la información genética entre generaciones? ¿A cuál de los integrantes de tu familia te pareces más? ¿Cómo lo justificarías?, -en el caso de las ideas previas de los estudiantes-. Con la información obtenida se realizó la descripción y/o caracterización del lugar y de la población con la que se implementaría la UD, así como el diseño de la misma.

Así pues, esta UD pretendía llevar a cabo en seis clases la explicación de conceptos como: Células Somáticas y Sexuales (participación de la mitosis y meiosis en los procesos de herencia), Los Cromosomas, El Gen, Estructura y Función del ADN, y Las Mutaciones. Sin embargo de las 6 clases planteadas sólo 4 se llevaron a cabo, por el inicio de actividades del colectivo docente en la institución-. La primera clase tenía por objeto reconocer la presencia de los cromosomas en todas las células, para ello se plantearon dos momentos, el primero fue el trabajo en dos grandes grupos, armando cada uno un rompecabezas de los procesos de Meiosis y Mitosis, considerando un debate posterior del tipo de células en cada proceso, lo que pasaba con los cromosomas en cada uno y la importancia de los mismos. El segundo momento fue la explicación de la estructura, la función y los tipos de los cromosomas, para lo cual se llevó a cabo una explicación teórica del tema. La segunda clase planteaba la observación de un video y una práctica de laboratorio en la que los estudiantes debían elaborar un pre informe planteando un anteproyecto (tipo de informe trabajado por la profesora). En este laboratorio se llevó a cabo la observación de células de raíz de cebolla en mitosis, identificando la presencia de cromosomas en metafase. La tercera clase consistió en la explicación de Genes dominantes y recesivos, a través de la elaboración de un árbol genealógico, del cual se tomaban ciertos caracteres, de acuerdo a los rasgos de los estudiantes. Al finalizar esta explicación se profundizó en los conceptos de Genotipo y Fenotipo, además se explicó el Cuadro de Punnett. En la cuarta clase se explicó el concepto de ADN, estructura, función, diferencias con el ARN, y se observaron 2 videos para clarificar conceptos. En la parte final de esta clase se hizo un cuadro comparativo entre ambos ácidos nucleicos, con las ideas que aportaban los estudiantes. La quinta clase comprendía la lectura de 8 noticias diversas de enfermedades (una para cada grupo). Posteriormente se llevaría a cabo un debate 
Edición Extraordinaria. p.p. 1625- 1635

Memorias del VIII Encuentro Nacional de Experiencias en Enseñanza de la Biología y la Educación Ambiental. III Congreso Nacional de Investigación en Enseñanza de la Biología.

entre los grupos, a partir del cual se explicaría en concepto de mutación y la influencia del ambiente en este proceso. La última clase consistía en la elaboración de una red conceptual por parte de los estudiantes, de manera individual. (Baracaldo Huertas \& Rincón García, 2015)

\section{RESULTADOS Y DISCUSIÓN:}

Los instrumentos utilizados fueron de gran ayuda para dar una idea general tanto del colegio, como del docente y el estudiante. Sin embargo, al aplicar el instrumento a los estudiantes estos se mostraron incomodos con el mismo, pues asumieron que serían evaluados, razón por la que se evidencio que contrario a lo que propone Cubero (1994), las preguntas fueron demasiado directas, por lo que queda el interrogante en cuanto a las ideas que los jóvenes plantearon, pues o fueron el resultado de la presion por sentirse evaluados, o la respuesta al interés por obtener una nota, cohibiendose de expresar sus verdaderas concepciones. En lo que se refiere al instrumento entregado a la profesora, ésta nunca devolvió el formato que le fue entregado.

En general, los estudiantes tuvieron una actitud participativa y se mostraron emocionados con las actividades propuestas, en ellos despertó gran interés realizar su árbol genealógico para identificar características heredadas a través de las generaciones, y las razones por las que se parecen más a uno de sus padres que al otro, e incluso hallaron más parecido con sus abuelos que con sus padres, surgiéndoles preguntas como: "¿Es verdad que los genes se saltan algunas generaciones?, ¿Por qué si mi abuela tiene ojos verdes, ni mi papá ni yo tenemos ojos claros?", hasta uno de los estudiantes preguntó si el SIDA era una enfermedad heredada genéticamente, y que se transmite sólo de madre a hijo. Justo cuando surgió esta pregunta la profesora irrumpe el transcurso de la clase para regañar al estudiante por introducir en la clase un tema que ya se había enseñado con antelación en la clase de sexualidad. Esta situación pone de presente una reflexión sobre el quehacer docente, pues como lo afirma Rodriguez de Moreno, Torres de Cárdenas, Franco Arbeláez, y Montañez Gómez, (2010) en la actualidad el docente se sale de casillas cuando surge un hecho sorpresivo, desaprovechando la oportunidad de educar a sus alumnos, lo que de cierta manera hace que el estudiante adquiera repulsion por realizar preguntas. 
Edición Extraordinaria. p.p. 1625- 1635

Memorias del VIII Encuentro Nacional de Experiencias en Enseñanza de la Biología y la Educación Ambiental. III Congreso Nacional de Investigación en Enseñanza de la Biología.

En cuanto al laboratorio, no hubo intéres por parte de todos los estudiantes, pues no hubo disponibilidad de materiales y esto generó desorden durante la primera hora de la clase. Al presentarse dicha situación la opción fue realizar la explicación teorica de lo que se esperaba observar; en este espacio los estudiantes realizaron preguntas en torno a la presencia de los cromosomas en la raíz y en las hojas de la planta, incluyendo preguntas acerca del uso del microscopio dentro de las que se destacó: "¿Para qué sirve la gota de agua cuando se pone la muestra en la lamina?(Estudiante de octavo, 2015), lo cual fue interesante, porque de alguna manera los estudiantes demostraron que estaban atentos a lo que se estaba explicando en la clase, promoviendo un proceso de reflexión con respecto a lo que puden aprender, tal como lo menciona Candela (2013):

(...) la enseñanza de las ciencias pretende que los alumnos piensen sobre lo que saben acerca de su realidad, que lo sepan exponer y que confronten sus explicaciones con las de sus compañeros, con la información que les da el maestro $u$ otros adultos y con lo que leen en los libros o reciben por diferentes medios. (Candela, 2013, pág. 2)

En el diseño de la UD hubo una incongruencia, pues se decidió enseñar a los estudiantes el cuadro de Punnett sin haberles enseñado las leyes de Mendel, ni enfatizado en los conceptos necesarios para que ellos comprendieran cuál era su utilidad en el campo de la genética. A pesar de que fue considerado un error en el diseño, sirvió para verificar lo señalado por Ayunso, Banet, y Abellán (1996) cuando refieren que los estudiantes sacan buenos resultados cuando resuelven los ejercicios propuestos en los libros de texto, pero cuando se les pide justificar la función de los resultados obtenidos, los estudiantes no logran explicar para qué se utiliza realmente el cuadro de Punnett, lo cual pone de presente que la realización de estos ejercicios se convierte en un procedimiento mecánico que da la ilusión de que el estudiante a comprendido el concepto cuando realmente no lo ha hecho.

En cuánto a las herramientas aplicadas en esta UD, el uso de materiales audiovisuales y de participación (como los rompecabezas, las preguntas, etc.) diferentes a la copia en el tablero y al uso de los libros de texto son de gran ayuda, ya que generan interés en el estudiante, además de constituir medios informativos atractivos para éste. 
Edición Extraordinaria. p.p. 1625- 1635

Memorias del VIII Encuentro Nacional de Experiencias en Enseñanza de la Biología y la Educación Ambiental. III Congreso Nacional de Investigación en Enseñanza de la Biología.

Finalmente, y a manera de reflexión, la implementación de esta UD pone de presente una de las tantas falencias actuales de la educación, la fragmentación del conocimiento, ya que ni el estudiante ni el docente establecen relaciones con los conocimientos adquiridos en otros campos del saber, y por ende al primero se le dificulta explicar, relacionar, e incluso comprender los contenidos enseñados en clase, y al segundo se le dificulta transformar las dinámicas tradicionales de la escuela, llegando en algunas ocasiones a subestimar la capacidad del estudiante para entender conceptos básicos como el de Proteína, por lo que el docente opta por cambiar de nombre para facilitar el aprendizaje, sin darse cuenta que esta generando un vacío en el conocimiento del estudiante, tal como lo menciona Rodríguez De Moreno, et. al. (2010) al mencionar que el maestro supone que cambiando los nombres relacionados con su práctica pedagógica ya demuestra una actualización y renovación en la forma de enseñar.

\section{CONCLUSIONES:}

Estas actividades ponen de presente la necesidad de integrar propuestas innovadoras a las dinámicas tradicionales en las aulas de clase, resaltando la importancia de las preconcepciones y del intercambio de ideas en la reestructuración de las concepciones para lograr la construcción de un conocimiento científico escolar, (García, 1997). También abren camino a nuevas investigaciones sobre la actitud actual de los docentes frente a la enseñanza de la genética y de las ciencias naturales.

\section{BIBLIOGRAFIA:}

ASTOLFI, J. P. (2001). Conceptos clave en la didáctica de las disciplinas. Sevilla: Diada editora. Serie Fundamentos No 17. Colección investigación y enseñanza.

AYUNSO, E., BANET, E., \& ABELLÁN, E. (1996). Introducción a la genética en la enseñanza secundaria y bachillerato: iresolución de problemas o realización de ejercicios? Enseñanza de las ciencias.

AYUNSO, G., \& BANET, E. (2002). Alternativas a la enseñanza de la genética en educación secundaria. Enseñanza de las ciencias, 133, 154.

BARACALDO HUERTAS, C. L., \& RINCÓN GARCÍA, D. C. (2015). Herencia genética: una mirada a través de lo que somos. Bogotá D.C. 
Bio - grafia. Escritos sobre la Biología y su Enseñanza. ISSN 2027

Edición Extraordinaria. p.p. 1625- 1635

Memorias del VIII Encuentro Nacional de Experiencias en Enseñanza de la Biología y la Educación Ambiental. III Congreso Nacional de Investigación en Enseñanza de la Biología.

BUGALLO RODRÍGUEZ, A. (1995). La didáctica de la genética: Revisión bibliografica. Enseñanza de las ciencias, 379-380.

CANDELA, M. A. (Agosto de 2013). Cómo se aprende y se puede enseñar ciencias naturales. Obtenido de Cómo se aprende y se puede enseñar ciencias naturales:

https://fcajuliocesar.files.wordpress.com/2013/08/canensenarciencias.doc

CUBERO, R. (1994). Conccepciones alternativas, preconceptos, errores conceptuales... ¿distinta terminologia y un mismo significado? Investigación en la escuela.

FINLEY, F., STEWART, J., \& YARROCH, W. (1982). Teacher's preparation of important and difficult science content: The report of a survey. Science Education, 531-538.

GARCÍA, E. (1997). La naturaleza del conocimiento escolar iTransición de lo cotidiano a lo cientifico, o de lo simple a lo complejo? En E. García, Conocimiento cotidiano, cientifico y escolar. (págs. 70-76). Sevilla.

JOHNSTONE, A. H., \& MAHMOUND, N. A. (1980). Isolating topics of high perceived difficulty in school biology. Journal of Biological Education., 163166.

POZO, I., \& GÓMEZ, M. (2004). Aprender y enseñar ciencia. Madrid: Morata.

RODRÍGUEZ DE MORENO, A., TORRES DE CÁRDENAS, R., FRANCO ARBELÁEZ, M. C., \& MONTAÑEZ GOMÉZ, G. (2010). EN BUSCA DE CONCEPTOS GEOGRÁFICOS. Bogotá: Universidad Pedagógica Nacional Fondo Editorial.

SMITH, M. U., \& SIMS, O. S. (1992). Cognitive development, genetics problem solving, and genetics instruction: A critical review. Journal of Research in Science Teaching, 895-912.

STEWART, J., \& KIRK, J. V. (1990). Understanding and problem-solving in classical genetics. International Journal of Science Education, 575-588. 
